\title{
Bouncing universe of entropy-corrected Friedmann equations
}

\author{
Amin Salehi ${ }^{a}$, Mojtaba Mahmoudi-Fard \\ Department of physics, Lorestan university, Khoramabad, Iran
}

Received: 20 January 2018 / Accepted: 11 March 2018 / Published online: 17 March 2018

(c) The Author(s) 2018

\begin{abstract}
In this paper, we investigate the possibility of obtaining bouncing-oscillating solution in modified Friedmann equations with logarithmic entropy corrected, $S=$ $\frac{A}{4 G}+\alpha \ln \frac{A}{4 G}+\beta \frac{4 G}{A}$, for positive, negative and zero values of $(\alpha, \beta)$ pre-factors and all kinds of curved universes. The results are argued using the dynamical system techniques and by employing the phase plane analysis for full classification of the nonsingular evolutions. Our analysis indicates that it is possible to have an oscillating universe as well as a bounce universe for $k=1$ and $k=-1$ curvatures. In $k=1$ case, both positive and negative values of $\alpha$ and $\beta$ can make bouncing-oscillating solution, while in $k=-1$ case, only the positive value of $\alpha$ with negative value of $\beta$ can make a bounce. Also the flat universe have no bounce solution.
\end{abstract}

\section{Introduction}

General relativity predicts a spacetime singularity for the beginning of the universe [1-4], as it is no longer valid and so there are some alternative theories to standard big bang cosmology [5-13]. Many attempts have been done to resolve this singularity problem through the modified general relativity theory [14-18], although it may be resolved via string theory and/or loop quantum cosmology $[19,20]$ as some candidates for a quantum theory of gravity [21-24]. In this right, the scenario of an oscillating universe is to avoid the big bang singularity and replace it with a cyclical evolution [25-38].

The quantum phenomenon of Hawking radiation [39] indicates that black hole has a temperature proportional to its surface gravity and an entropy proportional to its horizon area [39-42]. This issue led people to consider a profound connection between gravity and thermodynamics which was first addressed by Jacobson [43] who disclosed that the Einstein gravitational theory for the spacetime metric can be derived from the horizon entropy-area realation by using the

\footnotetext{
a e-mail: salehi.a@lu.ac.ir
}

fundamental Clausius relation, $\delta Q=T \delta S$, which is as the first law of thermodynamics for the cosmological horizon $[44,45]$. The investigations on the relation between Einstein field equations and first law of thermodynamics in the setup of black hole spacetime have been generalized to the cosmological context to derive Friedmann equations with any spatial curvature, by applying the Clausius relation to apparent horizon of the FRW universe [46-49]. See [50,51] for further studies of thermodynamical aspects of gravity, and also [52-56] as to refer to a recent review on possible cyclic models.

The so-called entropy-area formula which holds only in Einstein gravity has to be modified when some higher order curvature term appears in discussing the quantum correction to black hole entropy [57-62]. Therefore, the singularity problem - that comes from a "shortage of time" in the early universe - should be resolved and replaced by a quantum bounce $[63,64]$.

Inclusion of quantum effects, motivated from the loop quantum gravity (LQG) due to thermal equilibrium and quantum fluctuations [65-70], leads to the curvature correction in the Einstein-Hilbert action [71,72] provided as logarithmic entropy-corrected [66,73-76]

$S=\frac{A}{4 G}+\alpha \ln \frac{A}{4 G}+\beta \frac{4 G}{A}$

in entropy-area relationship of black holes in classical gravity, where $A=4 \pi \tilde{r}_{A}^{2}$ which $\tilde{r}_{A}=\frac{1}{\sqrt{H^{2}+\frac{k}{a^{2}}}}$ is the radius of apparent horizon, $\alpha$ and $\beta$ are dimensionless constants. The exact values of these constants are not yet determined and is still in debate within the quantum geometry (LQG). Various approaches to the black hole entropy yield the logarithmic correction involving $-\frac{1}{2}$ or $-\frac{3}{2}$ as popular values of $\alpha$ coefficient $[77,78]$. In these treatments, there is no such consensus with regard to how one might fix the value of the logarithmic pre-factor $\alpha$ (e.g. with $\beta=0$ ), as it appears to be a highly model dependent parameter [77-80]. 
Table 1 Number of critical points and existing bouncing-oscillating solutions for positive, negative and zero values of $(\alpha, \beta)$ pre-factors of entropy-corrected Friedmann equations describing the $k=1$ and $k=-1$ curved universes $\left(\alpha^{2}>12 \beta\right)$

\begin{tabular}{|c|c|c|c|c|c|}
\hline Model & $\alpha$ & $\beta$ & Number of critical points & Bounce & Oscillation \\
\hline $\mathrm{k}=1$ & Positive & Positive & 4 & Exist & Yes \\
\hline $\mathrm{k}=1$ & Positive & Negative & 2 & Exist & Yes \\
\hline $\mathrm{k}=1$ & Negative & Positive & 0 & Exist & No \\
\hline $\mathrm{k}=1$ & Negative & Negative & 2 & Exist & Yes \\
\hline $\mathrm{k}=1$ & 0 & 0 & 1 & Don't exist & No \\
\hline $\mathrm{k}=1$ & 0 & Negative & 2 & Exist & Yes \\
\hline $\mathrm{k}=1$ & 0 & Positive & 0 & Exist & No \\
\hline $\mathrm{k}=1$ & Positive & 0 & 2 & Exist & Yes \\
\hline $\mathrm{k}=1$ & Negative & 0 & 0 & Exist & No \\
\hline $\mathrm{k}=-1$ & Positive & Negative & 4 & Exist & Yes \\
\hline $\mathrm{k}=-1$ & Others & Others & 4 & Don't exist & No \\
\hline
\end{tabular}

Accordingly, for the time being the aim of this paper is twofold. The first is to see the importance of quantum effects on modification of Friedmann equations as to investigate the possibility of having solutions of the singularity-free cosmological model. The other is to evaluate the values of the constant pre-factors of Eq. (1) at bouncing evolution, corresponding to occurrence of bounce and having oscillating solutions, since they are not yet determined even within the loop quantum gravity. For instance, some works lead to the negative or positive $\alpha$ [77-86]; and the authour of [86] argued that $\alpha$ should be equal to zero.

The paper is organized as follows. In the next section we briefly discuss the modified Friedmann equations and bouncing approach by the action of the corrected entropyarea relation (1) to the apparent horizon of FRW universe. In Sect. 3 we get a spacetime of cosmological perturbations by studying the structure of the dynamical system via Jacobian stability combined with phase plane analysis. Some realizations of cosmological bounces and oscillating behaviors are discussed in Sect. 4. This situation, in general, is argued for all values of debated parameters $\alpha$ and $\beta$ by employing for different curvatures, as shown in Table 1 . We conclude in Sect. 5 with a discussion of approach and some key results for bouncing evolution of the entropy-corrected Friedmann equations on the metric of FRW universe.

\section{Modified Friedmann equations}

The Friedmann equations was derived by applying the first law of thermodynamics to the apparent horizon of the universe and assuming the geometric entropy given by a quarter of the horizon area [47]. However, addition to thermodynamical law of black hole's entropy as a dominant term given by a quarter of its horizon area, there is a quantum correction involving the logarithmic of the area [87]. On this basis, by tacking the corrected entropy-area relation (1) into account, the modified Friedmann equations of a FriedmannRobertson-Walker (FRW) universe with any spatial curvature, whose matter content constitutes a cold dark matter with the density $\rho$, would be $[48,49]$;

$$
\begin{aligned}
H^{2} & +\frac{k}{a^{2}}+\frac{\alpha G}{2 \pi}\left(H^{2}+\frac{k}{a^{2}}\right)^{2}-\frac{\beta G^{2}}{3 \pi^{2}}\left(H^{2}+\frac{k}{a^{2}}\right)^{3} \\
& =\frac{8 \pi G}{3} \rho \\
2 & \left(\dot{H}-\frac{k}{a^{2}}\right)\left(1+\frac{\alpha G}{\pi}\left(H^{2}+\frac{k}{a^{2}}\right)-\frac{\beta G^{2}}{\pi^{2}}\left(H^{2}+\frac{k}{a^{2}}\right)^{2}\right) \\
& =-8 \pi G(\rho+p),
\end{aligned}
$$

where we put $G=1$; and $k=0,1,-1$ represent a flat, closed and open universe, respectively. We also get $\frac{\alpha G}{\pi}$ as a new $\alpha$ and $\frac{\beta G^{2}}{\pi^{2}}$ as a new $\beta$ in the next sections of paper for simplicity. It is of interest to point out that there are different approaches to arrive at the same result, as the authors of $[88,89]$ have obtained the dynamical equations (1) and (2) by using the corresponding corrected entropy expression on the emergence spacetime that argued the accelerated expansion of the universe can be derived due to the difference between the surface degrees of freedom and the bulk ones in a region of space. Note that, given the recent astrophysical data [90-92], the universe is undergoing a state of accelerating expansion. We will thus suppose that dark energy might play an important role on the implications for the fate of the universe, and it's matter content is dominated by a dust matter (with the pressure $p=0$ ) at early time $[55,56]$.

Later on in the following sections within the dynamical calculation of these equations we shall see that the stability of nonsingular solutions occurs according to results summarized in Table 1 and depicted in phase plane portraits corresponding to particular regions/values of parameters $\alpha$ and $\beta$ for different curvatures of matter dominated universe. 


\section{Stability analysis}

In order to investigate bouncing evolution of universe, the stability structure of dynamical systems can be studied combined with phase plane analysis, by introducing the following new variables:

$\chi=H, \quad \zeta=a, \quad \eta=\rho$

From Eqs. (2) and (3), the evolution equations of these variables become,

$\dot{\chi}=-\frac{(1+\gamma) \eta}{2\left(1+2 \alpha^{\prime}\left(\chi^{2}+k \zeta^{-2}\right)-3 \beta\left(\chi^{2}+k \zeta^{-2}\right)^{2}\right)}+\frac{k}{\zeta^{2}}$

$\dot{\zeta}=\zeta \chi$

where a dot denotes a derivative with respect to the cosmic time. From Eq. (2), $\eta$ will be obtained as

$\eta=3\left(\chi^{2}+k \zeta^{-2}+\alpha\left(\chi^{2}+k \zeta^{-2}\right)^{2}-\beta\left(\chi^{2}+k \zeta^{-2}\right)^{3}\right)$

For next a bounce can also be defined locally. The minimal conditions from a local point of view for a bounce to happen in the case of a FLRW universe were analyzed in [93], where a Tolman wormhole was defined as a universe that undergoes a collapse, attains a minimum radius, and subsequently expands. Thus, to have a bounce it is necessary that $\dot{a}_{b}=0$ and $\ddot{a}_{b}>0$, or equivalently $\chi_{b}=0$ and $\left.\frac{d \chi}{d t}\right|_{t_{b}}>0$ are satisfied. For simplicity, we define $h_{b}=\left.\frac{d \chi}{d t}\right|_{t_{b}}$; as from Eq. (5)

$h_{b}=-\frac{k\left(\zeta_{b}^{4}-\alpha k \zeta_{b}^{2}+3 k^{2} \beta\right)}{2 \zeta_{b}^{2}\left(\zeta_{b}^{4}+2 \alpha k \zeta_{b}^{2}-3 k^{2} \beta\right)}$

Remembering that at bounce $H=0$, from Eq. (7), one can obtain the energy density at bounce, as

$\rho_{b}=\frac{3 k\left(\zeta_{b}^{4}+k \alpha \zeta_{b}^{2}-\beta k^{2}\right)}{\zeta_{b}^{6}}$

It is important to note that to have a bounce without violation of null energy condition, the following conditions must be satisfied.

$h_{b}>0$ and $\rho_{b}>0$

Note that, although the satisfaction of conditions (10) is necessary for a cyclic universe scenario in which the universe oscillates through a series of expansions and contractions, but it also needs more conditions from the dynamical system techniques.

Here, we employ the dynamical system technique to investigate when the system can oscillate, displaying that how a system behaves near a critical point. Under which condition the system can oscillate near the critical points. The phase plane analysis addresses the stability of solutions and trajectories of dynamical systems under small perturbations of initial conditions. Therefore, it can generally give us a full picture of the dynamics combined with phase plane analysis, especially in cosmology. Since such a study can be used to circumvent the need for initial conditions which is as one of the shortcomings of the standard cosmological model (SCM) [25-38,94]. In this right, the phase plane analysis is an invaluable tool in studying and visualizing the behavior of dynamical systems. The phase portraits in configuration space display the certain characteristics of system if the dynamics are stable or not. Hence one can know how trajectories behave near the critical points, e.g. whether they move toward or away from the fixed points [95].

As a well-studied problem, therefore, the stability of an orbit in phase space is reduced to a certain characteristics of system associated with the eigenvalues of Jacobian. In this case there are four critical points in the phase space, extreme points to describe the asymptotic behavior of the system, that are determined by simultaneously solving $\chi^{\prime}=0$ and $\zeta^{\prime}=0$.

$$
\begin{aligned}
& \chi_{1 c}=0, \quad \zeta_{1 c}=\sqrt{\frac{k}{2(1+3 \gamma)}}\left(-3 \gamma \alpha-\alpha+\left(9 \gamma^{2} \alpha^{2}\right.\right. \\
& \left.\left.-6 \gamma \alpha^{2}+\alpha^{2}-24 \gamma \beta-12 \beta+36 \gamma^{2} \beta\right)^{\frac{1}{2}}\right)^{\frac{1}{2}} \\
& \chi_{2 c}=0, \quad \zeta_{2 c}=-\sqrt{\frac{k}{2(1+3 \gamma)}}\left(-3 \gamma \alpha-\alpha+\left(9 \gamma^{2} \alpha^{2}\right.\right. \\
& \left.\left.-6 \gamma \alpha^{2}+\alpha^{2}-24 \gamma \beta-12 \beta+36 \gamma^{2} \beta\right)^{\frac{1}{2}}\right)^{\frac{1}{2}} \\
& \chi_{3 c}=0, \quad \zeta_{3 c}=\sqrt{\frac{-k}{2(1+3 \gamma)}}\left(-3 \gamma \alpha-\alpha+\left(9 \gamma^{2} \alpha^{2}\right.\right. \\
& \left.\left.-6 \gamma \alpha^{2}+\alpha^{2}-24 \gamma \beta-12 \beta+36 \gamma^{2} \beta\right)^{\frac{1}{2}}\right)^{\frac{1}{2}} \\
& \chi_{4 c}=0, \quad \zeta_{4 c}=-\sqrt{\frac{-k}{2(1+3 \gamma)}}\left(-3 \gamma \alpha-\alpha+\left(9 \gamma^{2} \alpha^{2}\right.\right. \\
& \left.\left.-6 \gamma \alpha^{2}+\alpha^{2}-24 \gamma \beta-12 \beta+36 \gamma^{2} \beta\right)^{\frac{1}{2}}\right)^{\frac{1}{2}}
\end{aligned}
$$

By virtue of this framework, we can study the stability analysis of critical points to investigate the properties of the dynamical system (Eqs. (5), (6)). Considering that a nonlinear autonomous system may be approximated by a linear system through its coefficient matrix (Jacobian), one can obtain the eigenvalues of Jacobian to standard classification of the different types of the fixed points [25-38]. In fact, the critical points of a system can be almost completely classified based on their eigenvalues, e.g. if they are complex, the point is called a focus and if system have eigenvalues with real part zero, the critical point is called center which is a neutrally stable closed orbit.

Here, the stability analysis is carried out for matter dominated model $\gamma=0$. Evaluating the Jacobian at the critical 
points gives us the eigenvalues of system to each of them as follows,

$\lambda_{ \pm}= \pm \frac{k \sqrt{3}}{3 \zeta_{c}\left(\alpha \zeta_{c}^{2}-2 \beta k\right)} \Omega^{\frac{1}{2}}$

where

$\Omega=-\alpha^{3} \zeta_{c}^{2}+3 k \alpha^{2} \beta+8 \alpha \zeta_{c}^{2} \beta-12 k \beta^{2}$

The parameter $\Omega$ is an important characteristic which its sign determines the nature of critical points and consequently explains the behavior of system near them. We shall obtain more insight into its dynamics in the next section.

\section{Oscillating evolution of curved universes}

In the following, we focus on matter dominated models to represent the oscillating evolution of different curved universes. The phase plane diagram of pertinent systems and in some cases the cyclic behavior of the scale factor are illustrated in the field plots of phase space, that gives us the opportunity to study all of the evolution paths admissible for all initial conditions. Therefore, the bouncing trajectories are shown in some detail and different oscillating solutions are obtained by tacking a certain condition for each portrait in the configuration space of this bouncing cosmology scenario.

\subsection{Case of spatially flat $(k=0)$ universe}

In the case of the cold dark matter dominated model, the system has an infinite number of critical points on $\chi=0$ axis. Also, there is four critical points on $\zeta=0$ line in phase space as follows:

$\zeta_{1 c}=0, \quad \chi_{1 c}=\frac{\sqrt{2}}{2 \beta} \sqrt{\beta\left(\alpha+\sqrt{\alpha^{2}+4 \beta}\right.}$

$\zeta_{2 c}=0, \quad \chi_{2 c}=-\frac{\sqrt{2}}{2 \beta} \sqrt{\beta\left(\alpha+\sqrt{\alpha^{2}+4 \beta}\right.}$

$\zeta_{3 c}=0, \quad \chi_{3 c}=\frac{\sqrt{2}}{2 \beta} \sqrt{\beta\left(\alpha-\sqrt{\alpha^{2}+4 \beta}\right.}$

$\zeta_{4 c}=0, \quad \chi_{4 c}=-\frac{\sqrt{2}}{2 \beta} \sqrt{\beta\left(\alpha-\sqrt{\alpha^{2}+4 \beta}\right.}$

All of the critical points on $\chi=0$ line, have the same eigenvalue as $\lambda_{ \pm}=(0,0)$. However, each of the critical points on $\zeta=0$ line, have the eigenvalue $\lambda_{ \pm}=\left(-3 \chi_{c}, \chi_{c}\right)$ in the phase space. Since the eigenvalues are real, the universe does not oscillate. Considering that the minimal conditions require a bounce with $\left(H_{b}=0, \dot{H}_{b}>0\right)$ that are evaluated at the bounce, the Eq. (3) implies that the bounce condition can not be satisfied. In fact, even if bounce occurs the null energy condition will be violated $(\rho+p)<0[96,97]$, so that in this case there is no bouncing solution.

\subsection{Case of hyperspherical $(k=1)$ universe}

In this case there are in general four critical points in the phase space as follows:

$$
\begin{aligned}
& P_{1}=\chi_{c}=0, \quad \zeta_{c}=\frac{\sqrt{2}}{2} \sqrt{\alpha+\sqrt{\alpha^{2}-12 \beta}}, \\
& P_{2}=\chi_{c}=0, \quad \zeta_{c}=-\frac{\sqrt{2}}{2} \sqrt{\alpha+\sqrt{\alpha^{2}-12 \beta}}, \\
& P_{3}=\chi_{c}=0, \quad \zeta_{c}=\frac{\sqrt{2}}{2} \sqrt{\alpha-\sqrt{\alpha^{2}-12 \beta}}, \\
& P_{4}=\chi_{c}=0, \quad \zeta_{c}=-\frac{\sqrt{2}}{2} \sqrt{\alpha-\sqrt{\alpha^{2}-12 \beta}},
\end{aligned}
$$

where they are mirror images of each other along the $\zeta=0$ line, considering that the condition $\alpha^{2}>12 \beta$ must be satisfied. The number of critical points and their properties are based on their eigenvalues, that they depend on the value of $\alpha$ and $\beta$ parameters. In the following, the respective classification of critical points in the phase space and corresponding eigenvalues are obtained for oscillating solutions accompanied by phase plane trajectories of system. Also, from Eqs. (8) and (9), $h_{b}$ and $\rho_{b}$ would be

$h_{b}=-\frac{\left(\zeta_{b}^{4}-\alpha \zeta_{b}^{2}+3 \beta\right)}{2 \zeta_{b}^{2}\left(\zeta_{b}^{4}+2 \alpha \zeta_{b}^{2}-3 \beta\right)}, \quad \rho_{b}=\frac{3\left(\zeta_{b}^{4}+\alpha \zeta_{b}^{2}-\beta\right)}{\zeta_{b}^{6}}$

\subsection{1 $k=1, \alpha=$ positive, $\beta=$ positive}

In this case $\alpha>\sqrt{\alpha^{2}-12 \beta}$ is preserved, so that through the condition $\alpha^{2}>12 \beta$, all of the four critical points exist. The eigenvalues of $P_{3}$ and $P_{4}$ are real with positive sign thus their nature is saddle-like, however the corresponding eigenvalues of $P_{1}$ and $P_{2}$ are purely imaginary. Therefore, the relation $\zeta_{c}^{2}=\frac{1}{2}\left(\alpha+\sqrt{\alpha^{2}-12 \beta}\right)$ holds for $P_{1}$ and $P_{2}$ points, and by substituting it in the Eq. (16), the parameter $\Omega$ will be

$\Omega=\left(-\frac{1}{2} \alpha^{4}-\frac{1}{2} \alpha^{3} \sqrt{\alpha^{2}-12 \beta}+4 \alpha \beta \sqrt{\alpha^{2}-12 \beta}+7 \alpha^{2} \beta-12 \beta^{2}\right)$

which is simplified as

$\Omega=\left(-\frac{1}{2}\left\{\left(\alpha^{2}-12 \beta\right)\left(\alpha^{2}-2 \beta\right)+\left(\alpha^{2}-8 \beta\right) \sqrt{\alpha^{2}-12 \beta}\right\}\right)$ 

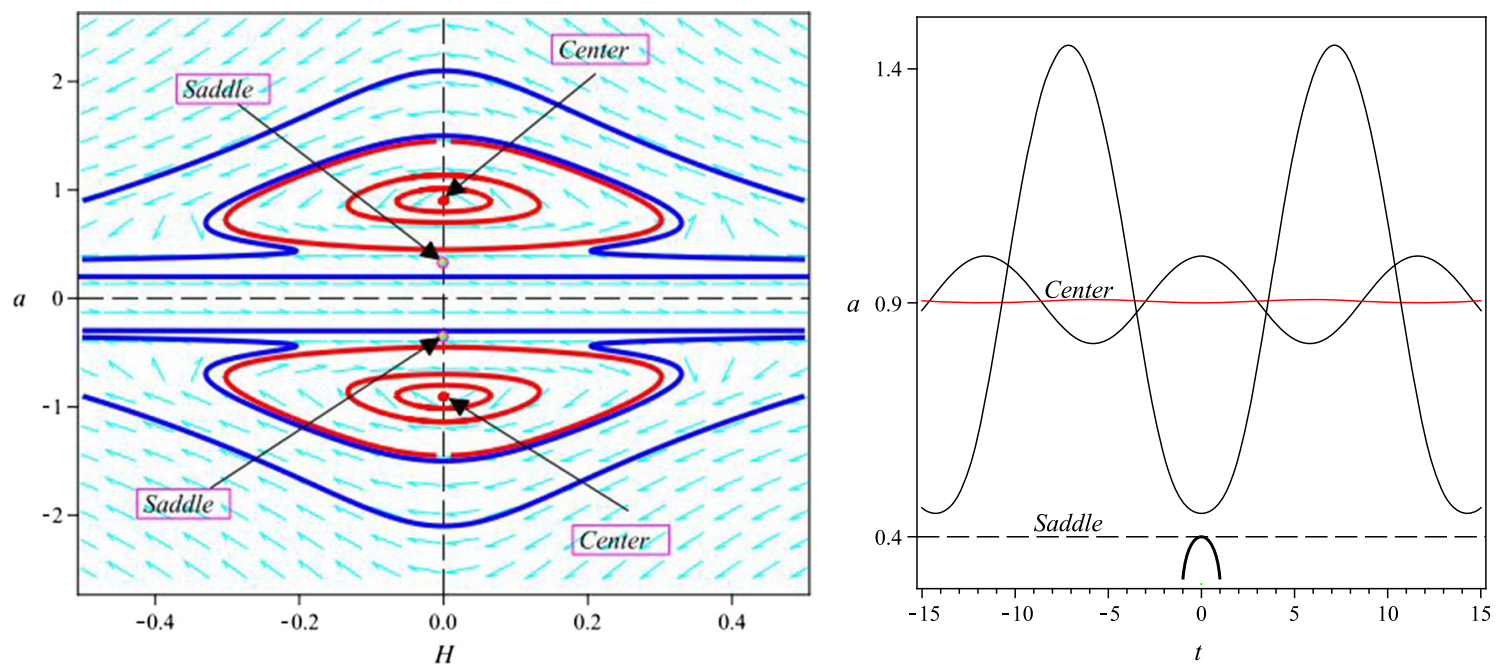

Fig. 1 (Left) dynamical behavior of the system around the critical points. (Right) time evolution of the scale factor $a$ corresponding to the trajectories in phase space for the case of $\gamma=0, k=1, \alpha=1$ and $\beta=0.05$
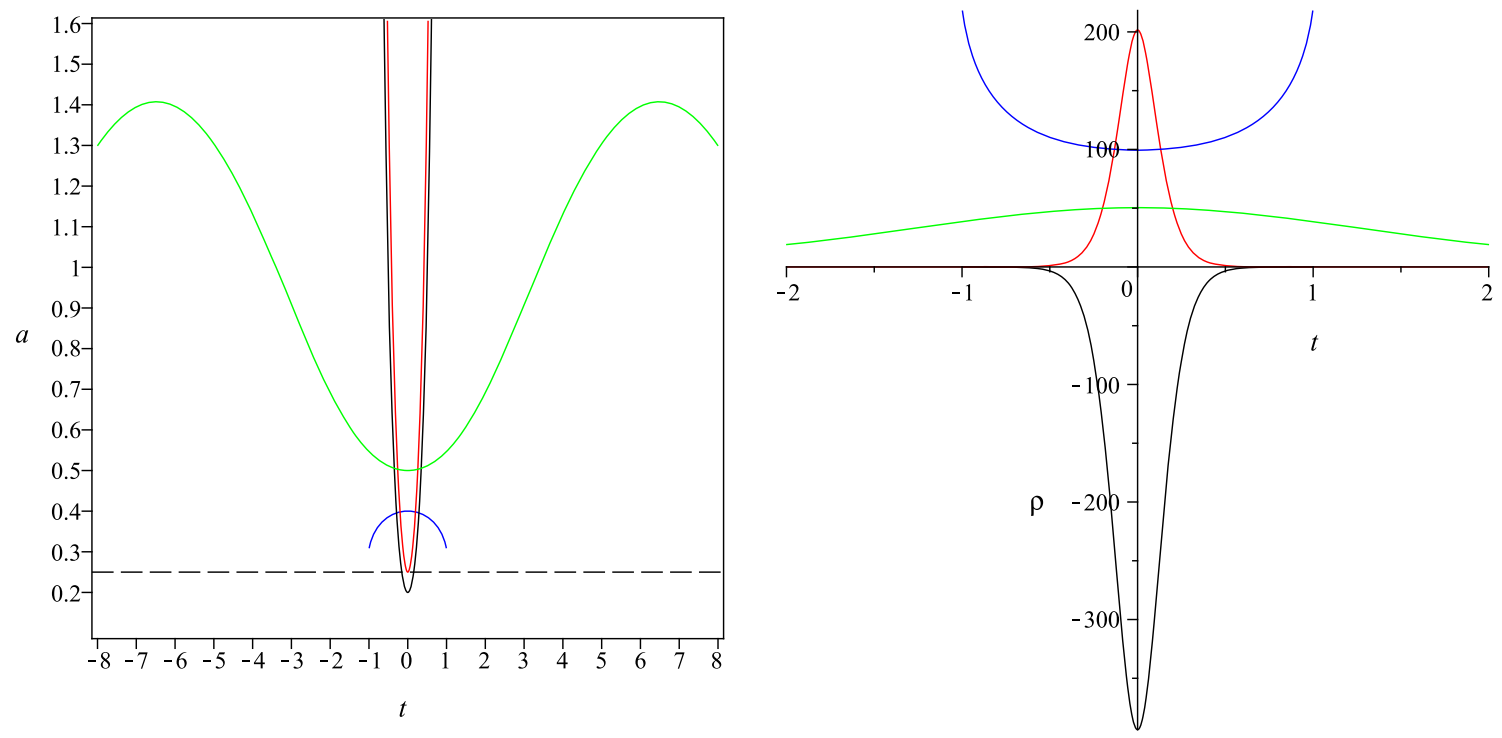

Fig. 2 Time evolution of the scale factor $a$ and energy density $\rho$, corresponding to the different initial conditions in phase space for the case of $\gamma=0, k=1, \alpha=1$ and $\beta=0.05$

For positive values of $\alpha$ and $\beta$ and via the condition $\left(\alpha^{2}-12 \beta>0\right)$, automatically the terms of $\left(\alpha^{2}-2 \beta\right)$ and $\left(\alpha^{2}-8 \beta\right)$ are positive. Therefore, $\Omega$ is negative and the eigenvalues are imaginary with zero real parts. This implies that the nature of the critical points $P_{1}$ and $P_{2}$ is center and marginally stable. The curves in the phase space are closed trajectories around the center. Interestingly, this behavior in phase space indicates that the scale factor of the universe undergoes contracting and expanding phases periodically, so that the universe can possess an exactly cyclic evolution. Also from Eq. (9), it directly follows that for positive values of $\alpha$ and $\beta$, the energy density would be positive at bounce only if $\zeta_{b}>\frac{1}{2} \sqrt{-2 \alpha+2 \sqrt{\alpha^{2}+4 \beta}}$

Thus, from all bouncing-oscillating trajectories of the phase space, those trajectories which satisfy the condition (24) are admissible solutions. To clarify this, we have plotted the phase space $a-H$ for $\alpha=1$ and $\beta=0.05$ (Fig. 1). Also, the evolution of scale factor and energy density as a function of time for important trajectories have been shown in Fig. 2. By assuming $\alpha=1$ and $\beta=0.05$, the four critical points would be

$$
\begin{aligned}
& P_{1}=(\chi=0, \zeta=0.9), \quad P_{2}=(\chi=0, \zeta=-0.9), \\
& P_{3}=(\chi=0, \zeta=0.4), \quad P_{4}:(\chi=0, \zeta=-0.4)
\end{aligned}
$$



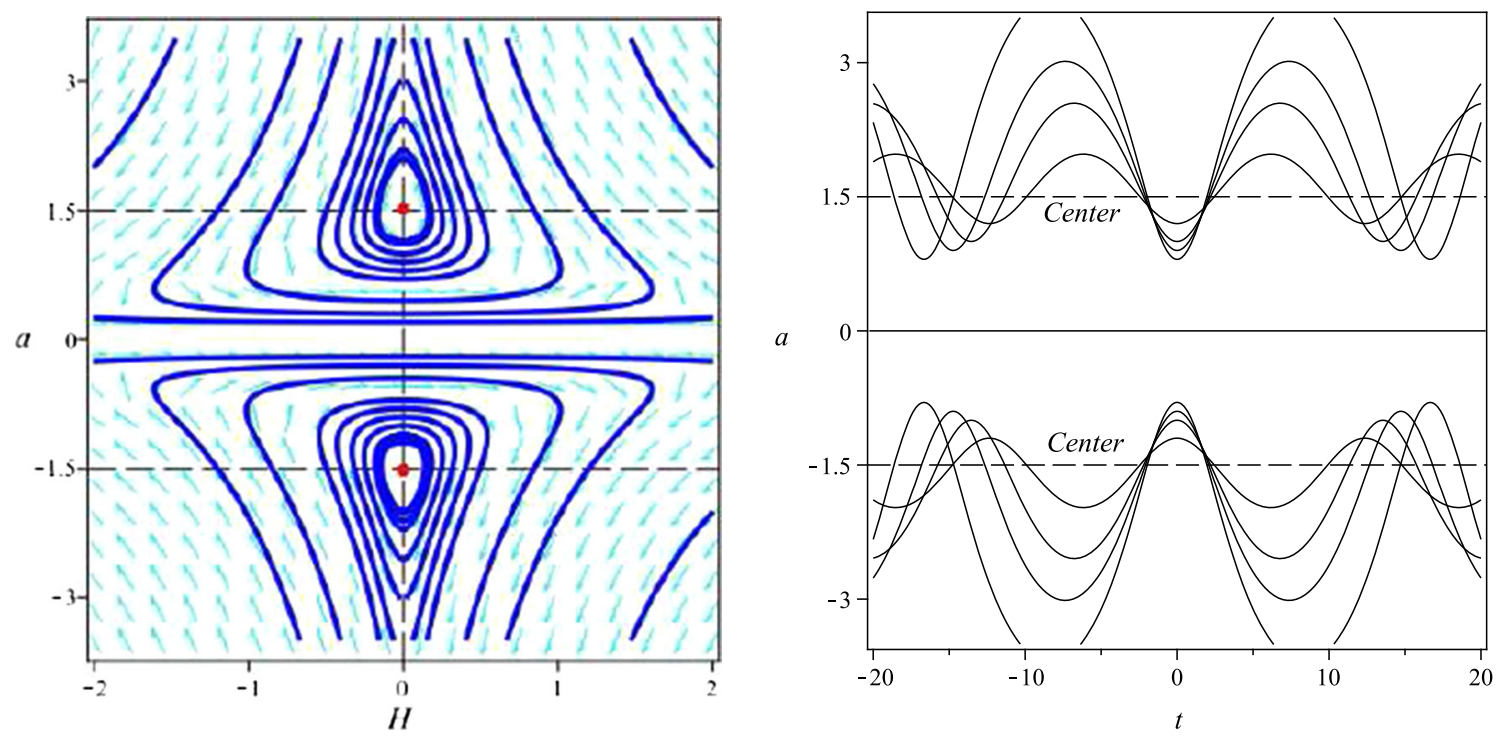

Fig. 3 (Left) dynamical behavior of the system around the critical points. (Right) time evolution of the scale factor $a$ corresponding to the trajectories in phase space for the case of $\gamma=0, k=1, \alpha=1$ and $\beta=-1$

By evaluating the Jacobian at the critical points, we get

$\lambda_{1}=(0.5 I, \zeta=-0.5 I), \quad \lambda_{2}=(0.5 I, \zeta=-0.5 I)$,

$\lambda_{3}=(1.6,-1.6), \quad \lambda_{4}=(1.6,-1.6)$

These are corresponding eigenvalues, as the stable critical points $P_{1}$ and $P_{2}$ are called center while $P_{3}$ and $P_{4}$ are unstable fixed points or singular saddle points. It would be interesting to see whether the initial conditions are important in cosmological dynamics systems. From Eq. (24) for $\alpha=1$ and $\beta=0.05$, if $a_{b}=\zeta_{b}>0.23$, one can see that the energy density will then be positive. Figure 2 also illustrates the point that the two curves (red and blue), where both have approximately the same bounce but a little difference in initial conditions, have made completely different evolution in their energy density. The red curve, with positive energy density which $a_{b}=\zeta_{b}>0.23$, satisfies the condition (24), while the blue curve $a_{b}=\zeta_{b}<0.23$ does not.

\subsection{2 $k=1, \alpha=$ positive, $\beta=$ negative}

For positive $\alpha$ and negative $\beta, \alpha<\sqrt{\alpha^{2}-12 \beta}$ is preserved, and the terms of $\left(\alpha^{2}-12 \beta\right),\left(\alpha^{2}-2 \beta\right)$ and $\left(\alpha^{2}-8 \beta\right)$ are positive. Therefore, there are only two of the above critical points, $P_{1}$ and $P_{2}$, which again are center. The corresponding eigenvalues are also purely imaginary and the universe has an oscillating behavior in the phase space. As shown in Fig. 3, for example, by setting $\alpha=1$ and $\beta=-1$, the two critical points would be $P_{1}=(\chi=0, \zeta=1.5)$ and $P_{2}=(\chi=0, \zeta=-1.5)$ with the same eigenvalue $\lambda_{1,2}=(0.5 I,-0.5 I)$

One may note that from Eq. (24), the energy density at bounce point in this case is positive.

\subsection{3 $k=1, \alpha=$ negative, $\beta=$ positive}

Since $\alpha$ is negative and $\beta$ is positive, the expressions $\alpha-$ $\sqrt{\alpha^{2}-12 \beta}<0$ and $\alpha+\sqrt{\alpha^{2}-12 \beta}<0$ are established; so that the system does not have any real critical point and consequently the oscillating solutions do not exist. However, a single bounce without oscillation can occur under the appropriate conditions. This is possible to make by satisfaction of minimal requirement of bounce condition $\left(H_{b}=0\right.$ and $\dot{H}_{b}>0$ ), and it can also follow equivalently at the bounce as $\left.\frac{d \chi}{d t}\right|_{t_{b}}>0$ in terms of new variables.

In order to explain this more fully, it is useful to obtain the energy density and $\left(\frac{d \chi}{d t}\right)$ at bounce. From Eq. (7), the energy density would be

$\rho_{b}=\frac{3\left(\zeta_{b}^{4}+\alpha \zeta_{b}^{2}-\beta\right)}{\zeta_{b}^{6}}$

This relation indicates that only under the condition $\left(\zeta_{b}>\frac{\sqrt{2}}{2} \sqrt{-\alpha+\sqrt{\alpha^{2}+4 \beta}}\right)$, the energy density will be positive. Also, from Eq. (5)

$h_{b}=\left.\frac{d \chi}{d t}\right|_{t_{b}}=-\frac{\left(\zeta_{b}^{4}-\alpha \zeta_{b}^{2}+3 \beta\right)}{2 \zeta_{b}^{2}\left(\zeta_{b}^{4}+2 \alpha \zeta_{b}^{2}-3 \beta\right)}$

Since the positive energy density automatically implies the positive $\left(\zeta_{b}^{4}+\alpha \zeta_{b}^{2}-\beta\right)$, the following inequality holds for this case

$\zeta_{b}^{4}-\alpha \zeta_{b}^{2}+3 \beta>\left(\zeta_{b}^{4}+\alpha \zeta_{b}^{2}-\beta\right)$ 

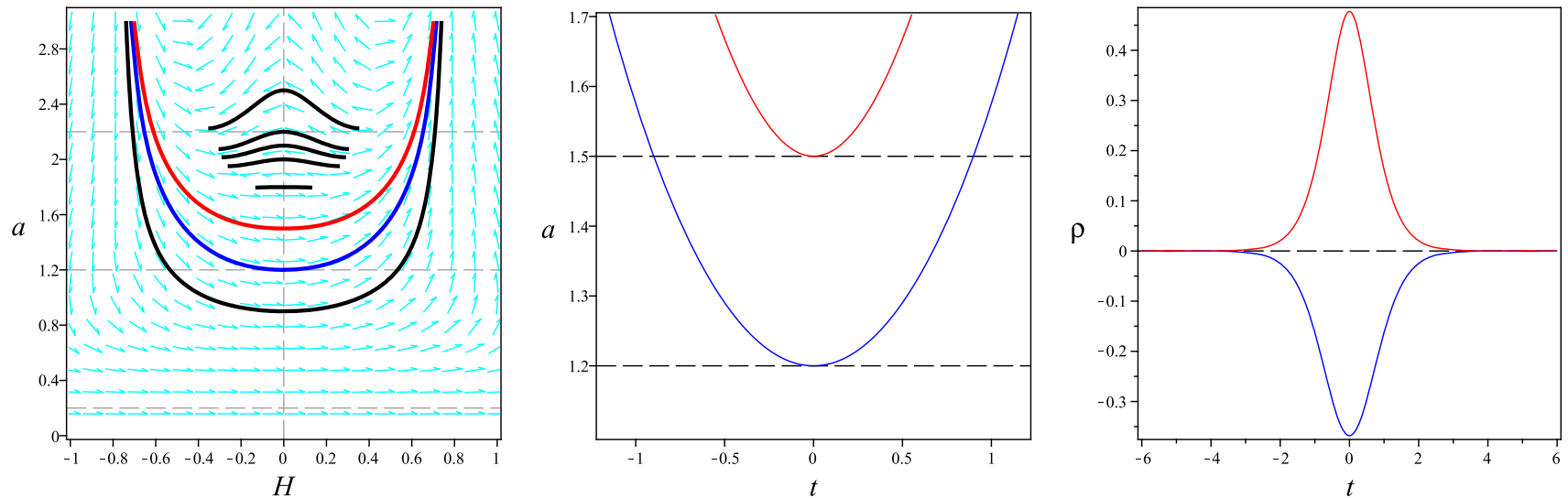

Fig. 4 (Left) dynamical behavior of the system around the critical points (Middle) time evolution of the scale factor corresponding to the red and blue trajectories. (Right) time evolution of the energy density corresponding to these trajectories

Therefore, the positive energy density naturally implies the positive $\zeta_{b}^{4}-\alpha \zeta_{b}^{2}+3 \beta$, as from Eq. (26), the bounce would be occur only for negative $\zeta_{b}^{4}+2 \alpha \zeta_{b}^{2}-3 \beta$ or equivalently when $\left(\zeta_{b}<\sqrt{-\alpha+\sqrt{\alpha^{2}+3 \beta}}\right)$. We can conclude that under following condition

$$
\left(\frac{\sqrt{2}}{2} \sqrt{-\alpha+\sqrt{\alpha^{2}+4 \beta}}\right)<\zeta_{b}<\left(\sqrt{-\alpha+\sqrt{\alpha^{2}+3 \beta}}\right)
$$

the bounce has occurred and energy density will be positive. The phase plane diagrams for the case of $\alpha=-1$ and $\beta=1$ have been plotted in Fig. 4 for clarifying this point, as the evolution of scale factor and energy density have also been shown for different initial conditions $\zeta_{b}$. In this right, we have $\frac{\sqrt{2}}{2} \sqrt{1+\sqrt{5}} \simeq 1.27<\zeta_{b}<\sqrt{3} \simeq 1.7$ to avoid a negative energy density at bounce, as there exists bouncing solution with positive energy density. Note that, it is possible to have a bounce while the energy density is negative only when the r.h.s of above inequality (28) is preserved.

\subsection{4 $k=1, \alpha=$ negative, $\beta=$ negative}

For this case as in the case of $\alpha>0, \beta<0$, there are only two critical points, $P_{1}$ and $P_{2}$. The three dimensional plot of $\Omega$ as a function of $\alpha$ and $\beta$ has been drawn in Fig. 5, which in some regions is positive and (in other parts) negative. Hence the eigenvalues of the system can be real or complex, as in those regions that $\Omega$ is negative the universe has oscillating solution and trajectories are closed orbits in the phase space.

For instance, let us get $\alpha=-0.1$ and $\beta=-0.1$ in accordance with Fig. 6. Hence, the value of $\Omega$ is negative and pertinent eigenvalues are purely imaginary, as the trajectories in phase plane are closed curves near the critical points. These points have the characteristics of a center fixed point and

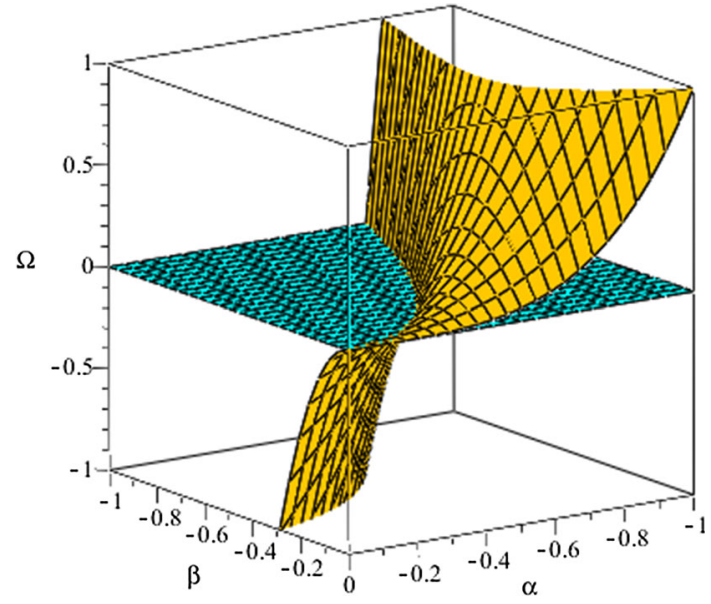

Fig. 5 The three dimensional plot of $\Omega$ as a function of $\alpha$ and $\beta$ for $k=1$

system oscillates around them. The energy density would be positive if the condition $\left(\zeta_{b}>\frac{\sqrt{2}}{2} \sqrt{-\alpha+\sqrt{\alpha^{2}+4 \beta}}\right)$ is satisfied, similar to what was found for the case of positive $\alpha$ and positive $\beta$.

\section{$4.2 .5 k=1, \alpha=0, \beta=0$}

The system has one critical point $\left(\chi_{c}=0, \zeta_{c}=0\right)$ and its eigenvalues are $\lambda_{ \pm}=0$, so that the universe does not oscillate. Applying Eq. (2) into the (3) yields

$2 \dot{H}+3 H^{2}=-\frac{k}{a^{2}}$

So $\dot{H}$ is negative, meaning that the universe does not oscillate and bounce never occurs. In this case the universe expands and reaches to its maximum size and then collapses. Consequently, the energy density decreases during the expansion and it reaches to its minimum values then increases. The 


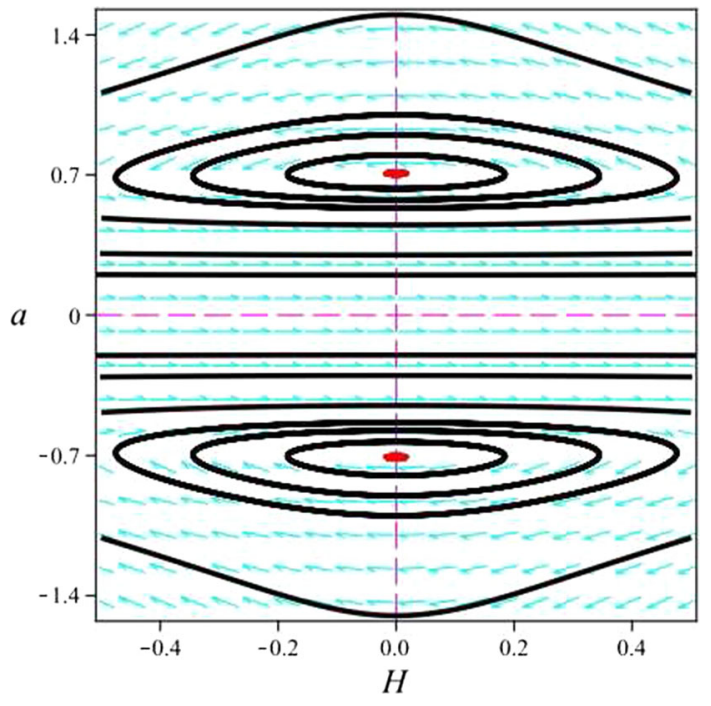

Fig. 6 Phase plane of parameters $(a, H)$ for the case of $k=1, \alpha=$ -0.1 , and $\beta=-0.1$

two dimensional phase space of $(a, H)$ and time evolution of scale factor have been shown in Fig. 7.

\subsection{6 $k=1, \alpha=$ positive, $\beta=0$}

For this case the system has two critical points $\left(\chi_{c}=0, \zeta_{c}=\right.$ $\sqrt{\alpha})$ and $\left(\chi_{c}=0, \zeta_{c}=-\sqrt{\alpha}\right)$, and corresponding eigenvalues are $\lambda_{1}=\left(\frac{i \sqrt{3}}{3 \sqrt{\alpha}}, \frac{-i \sqrt{3}}{3 \sqrt{\alpha}}\right)$ and $\lambda_{2}=\left(\frac{i \sqrt{3}}{3 \sqrt{\alpha}}, \frac{-i \sqrt{3}}{3 \alpha}\right)$ which are the same. The eigenvalues are purely imaginary and conjugated, as the critical points are centers (nonhyperbolic critical points) with closed curves that are turned around them. This evolution predicts a cyclic universe, with positive energy

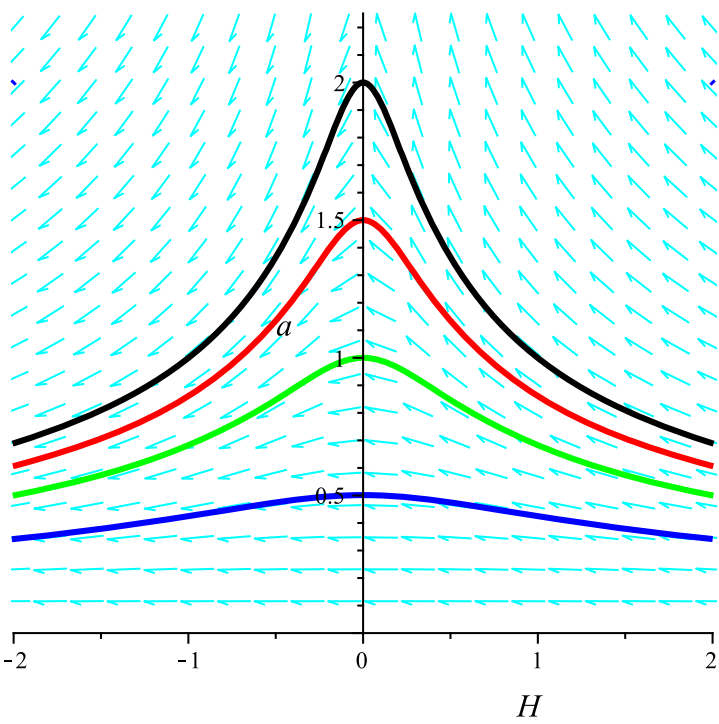

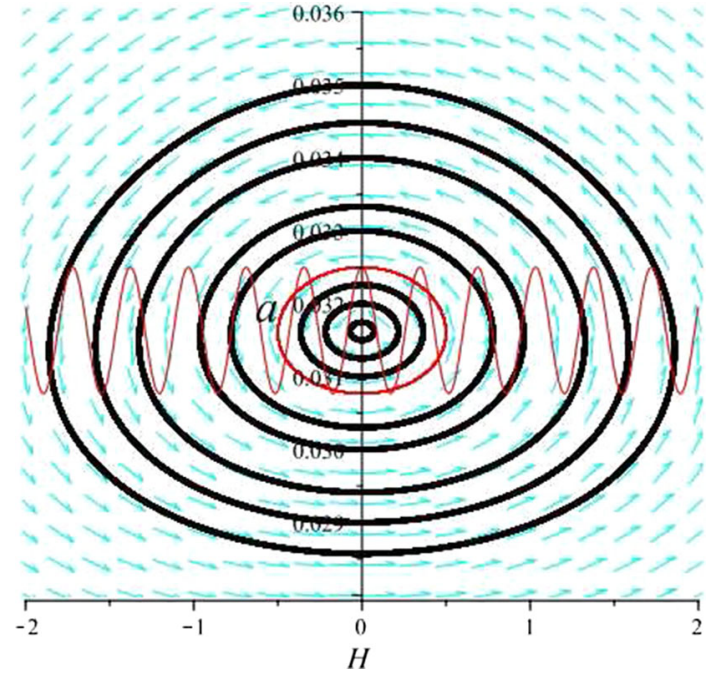

Fig. 8 Phase space of the dynamical system for the case of $k=1, \alpha=$ $0.001, \beta=0$. The dynamical behavior of the scale factor for an arbitrary trajectory of phase space (red trajectory) has been plotted

density at bounce from the Eq. (9), where the minimal and maximal values of the scale factor remain the same in every cycle (see Fig. 8).

\subsection{7 $k=1, \alpha=$ negative, $\beta=0$}

In this case, the system doesn't have any real critical point and it doesn't oscillate. However, minimal condition for a bounce $\chi_{b}=0$ and $\left.\frac{d \chi}{d t}\right|_{t_{b}}>0$ may be satisfied. If we put $\chi=0$ in the r.h.s of Eq. (5), the expression $\frac{d \chi}{d t}=-\frac{\zeta^{2}-\alpha}{\zeta^{2}\left(\zeta^{2}+2 \alpha\right)}$ is obtained. This condition denotes that for $\zeta_{b}=a_{b}<\sqrt{-2 \alpha}$,

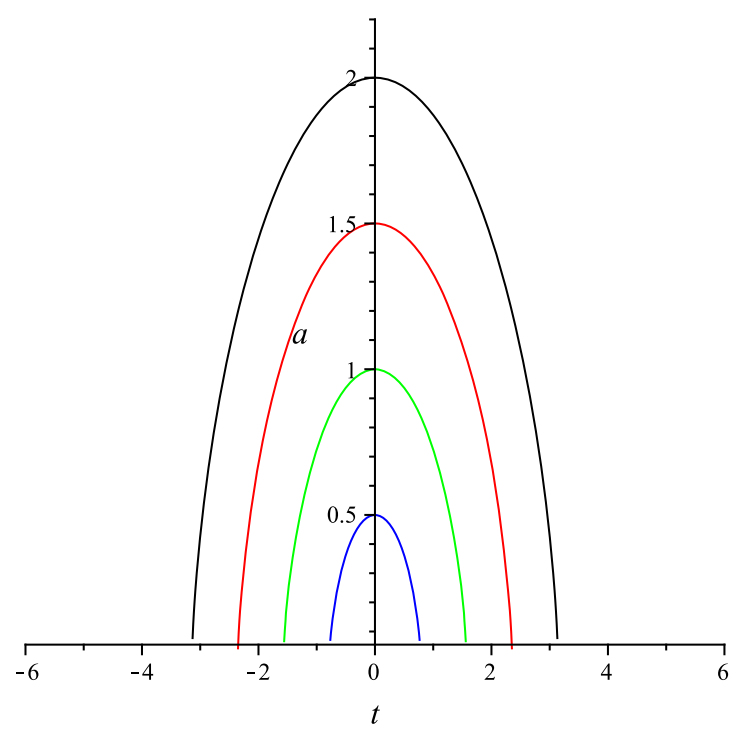

Fig. 7 (Left) phase plane of parameters $(a, H)$ for the case of $k=1, \alpha=0, \beta=0$. (Right) time evolution of the scale factor corresponding to the highlighted trajectories of the phase space 

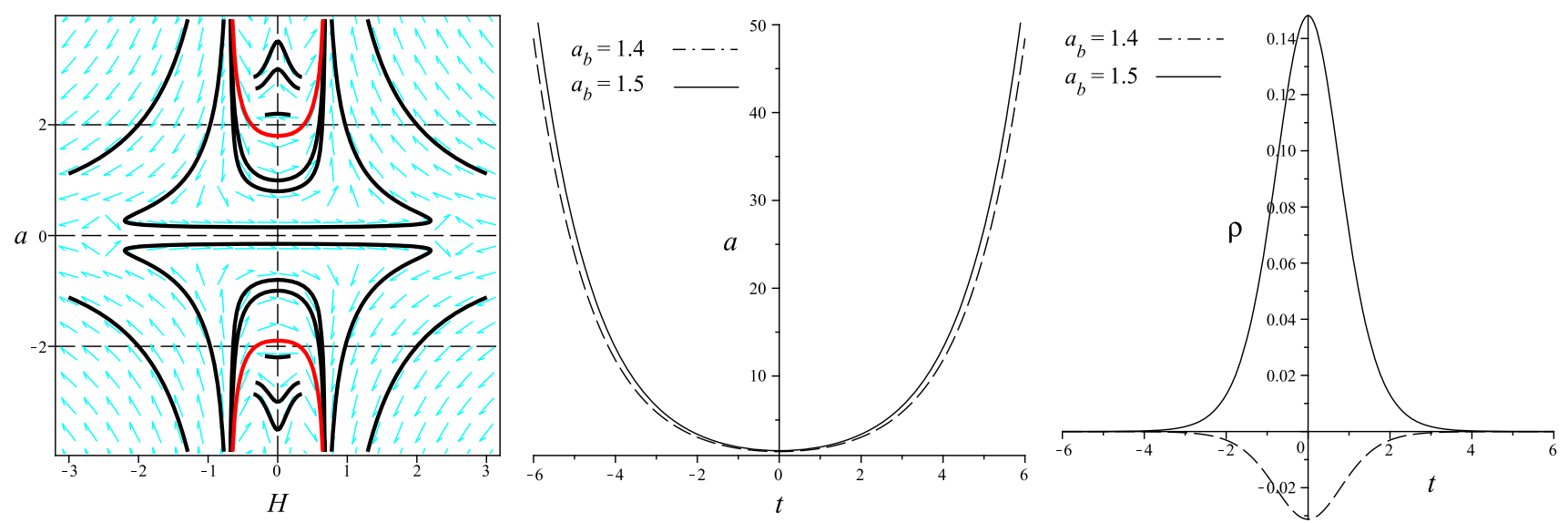

Fig. 9 Phase space of the dynamical system for the case of $k=1, \alpha=-2, \beta=0$

the bounce can occur and from Eq. (9) the energy density will be positive. In order to illustrate this matter in phase space configuration, by setting $\alpha=-2$ as shown in Fig. 9, the bounce take places for $a_{b}<2$ whereas for $a_{b}>2$ the bounce backs or collapses.

\subsection{8 $k=1, \alpha=0, \beta=$ positive}

In this case, the system does not have any real critical point and does not oscillate. However, minimal condition for a bounce may be satisfied. In this regard, given the positive $\rho_{b}=\frac{3\left(\zeta_{b}^{4}-\beta\right)}{\zeta_{b}^{6}}$ and $\left.\frac{d \chi}{d t}\right|_{t_{b}}=-\frac{\left(\zeta_{b}^{4}+3 \beta\right)}{2 \zeta_{b}^{2}\left(\zeta_{b}^{4}-3 \beta\right)}$, and hence $\beta^{\frac{1}{4}}<$ $\zeta_{b}<(3 \beta)^{\frac{1}{4}}$, which may be obtained by setting $\alpha=0$ in Eq. (28), there is a single bounce with positive energy density.

\subsection{9 $k=1, \alpha=0, \beta=$ negative}

In this case, the system has two real critical points as $\left(\chi_{c}=\right.$ $\left.0, \zeta_{c}=(-3 \beta)^{\frac{1}{4}}\right)$ and $\left(\chi_{c}=0, \zeta_{c}=-(-3 \beta)^{\frac{1}{4}}\right)$. The corresponding eigenvalues are $\lambda_{1}=\left(\frac{i}{\zeta_{c}}, \frac{-i}{\zeta_{c}}\right)$ and $\lambda_{2}=\left(\frac{i}{\zeta_{c}}, \frac{-i}{\zeta_{c}}\right)$ which are the same. Since the eigenvalues are purely imaginary and conjugated, the closed trajectories of configuration space turn around the nonhyperbolic critical points (centers). The phase plane diagram in Fig. 10 is to illustrate the situation which simplifies the understanding of system as describing the dynamics of cosmological parameters (scale factor and Hubble parameter) to provide a more meaningful insight on the setting of initial conditions.

\subsection{Case of hyperbolic $(k=-1)$ universe}

By inclusion of a negative curvature index $k=-1$ in modified Friedmann equations ((2), (3)), the number of critical points are as follows

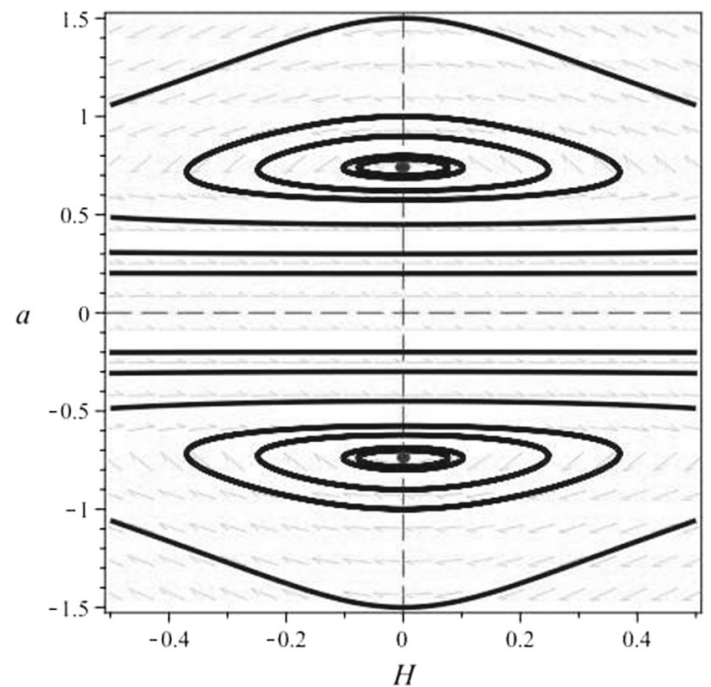

Fig. 10 The two dimensional phase plane for parameters $(a, H)$, by setting $\alpha=0, \beta=-0.1$

$$
\begin{aligned}
& P_{1}=\chi_{c}=0, \quad \zeta_{c}=\frac{\sqrt{2}}{2} \sqrt{-\alpha+\sqrt{\alpha^{2}-12 \beta}} \\
& P_{2}=\chi_{c}=0, \quad \zeta_{c}=-\frac{\sqrt{2}}{2} \sqrt{-\alpha+\sqrt{\alpha^{2}-12 \beta}} \\
& P_{3}=\chi_{c}=0, \quad \zeta_{c}=\frac{\sqrt{2}}{2} \sqrt{-\alpha-\sqrt{\alpha^{2}-12 \beta}} \\
& P_{4}=\chi_{c}=0, \quad \zeta_{c}=-\frac{\sqrt{2}}{2} \sqrt{-\alpha-\sqrt{\alpha^{2}-12 \beta}}
\end{aligned}
$$

Also,

$\rho_{b}=-\frac{3\left(\zeta_{b}^{4}-\alpha \zeta_{b}^{2}-\beta\right)}{\zeta_{b}^{6}}, \quad h_{b}=\frac{\left(\zeta_{b}^{4}+\alpha \zeta_{b}^{2}+3 \beta\right)}{2 \zeta_{b}^{2}\left(\zeta_{b}^{4}-2 \alpha \zeta_{b}^{2}-3 \beta\right)}$ 


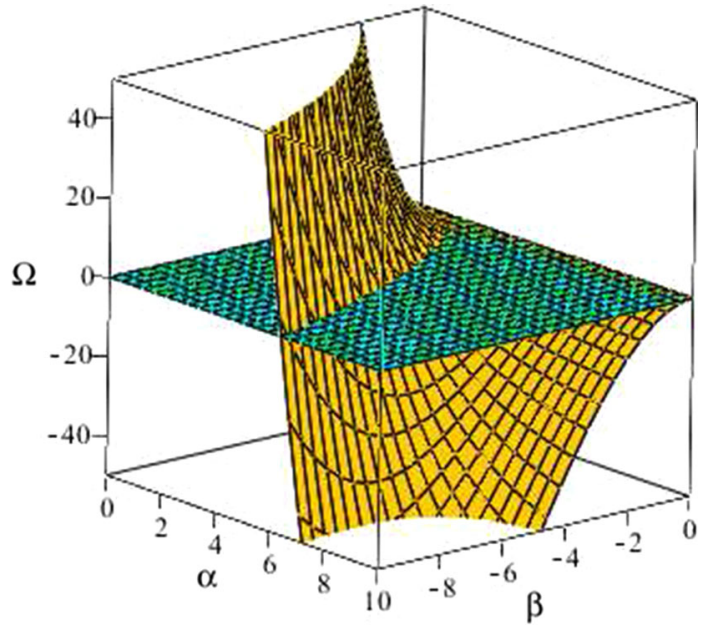

Fig. 11 The three dimensional plot of $\Omega$ as a function of $\alpha$ and $\beta$ for $k=-1$

Given the minimal condition that a bounce needs to occur from the local point of view while the energy density is positive $\left(h_{b}>0, \rho_{b}>0\right)$, the various possibilities to satisfy this condition can be classified as

$\left\{\begin{array}{l}\rho_{b}>0 \Longrightarrow\left(\zeta_{b}^{4}-\alpha \zeta_{b}^{2}-\beta\right)<0 \\ h_{b}>0 \Longrightarrow\left\{\begin{array}{l}\left(\zeta_{b}^{4}+\alpha \zeta_{b}^{2}+3 \beta\right)>0,\left(\zeta_{b}^{4}-2 \alpha \zeta_{b}^{2}-3 \beta\right)>0 \\ \left(\zeta_{b}^{4}+\alpha \zeta_{b}^{2}+3 \beta\right)<0,\left(\zeta_{b}^{4}-2 \alpha \zeta_{b}^{2}-3 \beta\right)<0\end{array}\right.\end{array}\right.$

The above classification can be rearranged as two sets of following conditions,

CI $:\left\{\begin{array}{l}i:-\left(\zeta_{b}^{4}-\alpha \zeta_{b}^{2}-\beta\right)>0 \\ i i:\left(\zeta_{b}^{4}+\alpha \zeta_{b}^{2}+3 \beta\right)>0 \\ i i i:\left(\zeta_{b}^{4}-2 \alpha \zeta_{b}^{2}-3 \beta\right)>0\end{array} \quad\right.$ CII: $\left\{\begin{array}{l}i:\left(\zeta_{b}^{4}-\alpha \zeta_{b}^{2}-\beta\right)<0 \\ i i:\left(\zeta_{b}^{4}+\alpha \zeta_{b}^{2}+3 \beta\right)<0 \\ i i i:\left(\zeta_{b}^{4}-2 \alpha \zeta_{b}^{2}-3 \beta\right)<0\end{array}\right.$

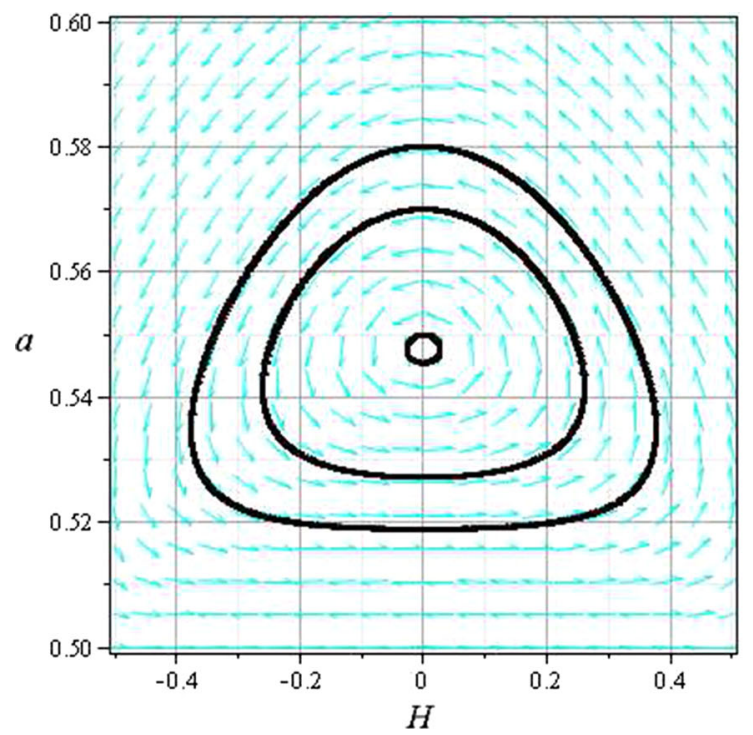

All of the (i), (ii) and (iii) conditions of CI class or all of them for CII class should be satisfied to have a bounce. The combining of (i) and (ii) conditions of CI, gives the $\left(2 \alpha \zeta_{b}^{2}+4 \beta\right)>0$ condition which is in contradiction with the result of the combined provision of the (i) and (iii) conditions, i.e. $\left(2 \alpha \zeta_{b}^{2}+4 \beta\right)<0$. However, a similar calculation on the (i), (ii) and (iii) conditions of CII shows that it would be satisfied only if $\alpha>0$ and $\beta<0$. One can conclude, therefore, that there is the possibility of having the oscillating solution only for positive $\alpha$ and negative $\beta$ of a negative curvature $k=-1$ universe.

\subsection{1 $k=-1, \alpha=$ positive, $\beta=$ negative}

For positive $\alpha$ and negative $\beta$, the expression $\alpha^{2}-12 \beta>0$ and hence $\left(-\alpha+\sqrt{\alpha^{2}-12 \beta}\right)$ are positive while $(-\alpha-$ $\sqrt{\alpha^{2}-12 \beta}$ ) is negative. Consequently, there are only two of the above mentioned critical points, $P_{1}$ and $P_{2}$. The eigenvalues of the system can be real or complex. The three dimensional plot of $\Omega$, shown in Fig. 11 as a function of $\alpha$ and $\beta$, illustrates the positive and negative values of $\Omega$ in different regions. For example by setting $\alpha=0.7$ and $\beta=-0.1$, the eigenvalues of system would be $\lambda= \pm(6.6 I,-6.6 I)$ (Fig. 12), whereas for $\alpha=0.3$ and $\beta=-0.1$ the eigenvalues are real as $\lambda= \pm(2.3,-2.3)$. Therefore, it is possible to have a bouncing-oscillating solution by proper choosing of $\alpha$ and $\beta$.

\subsection{2 $k=-1$, Othercases}

As it was mentioned above, other cases of a negative curvature $k=-1$ universe have no oscillating solution. The formal analysis appropriately can be applied the same as expres-

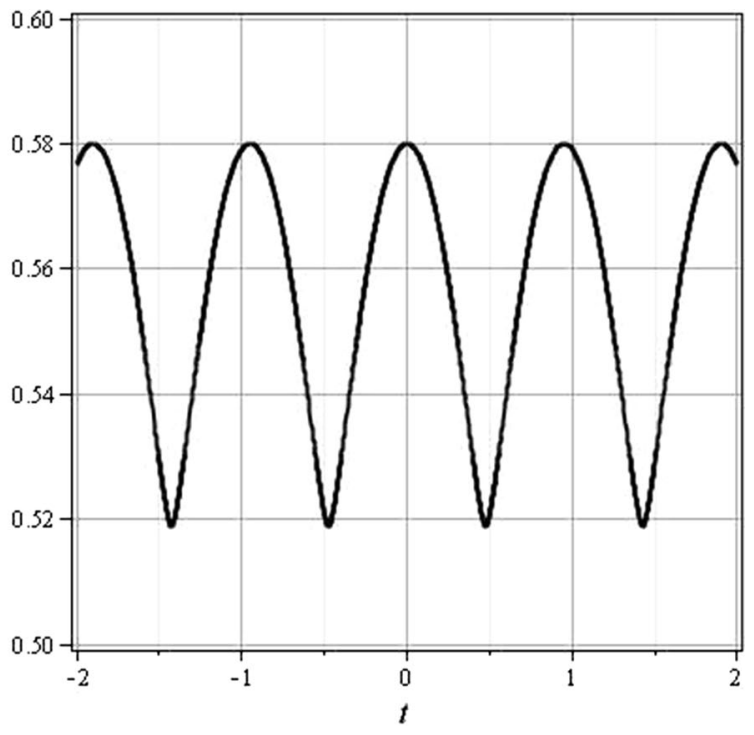

Fig. 12 The two dimensional phase plane for parameters $(a, H)$. We have set $k=-1, \alpha=0.7, \beta=-0.1$ 
sions of a positive curvature universe. Let us consider the case of $\alpha=$ positive and $\beta=$ positive, for example, where both the values of $\left(-\alpha-\sqrt{\alpha^{2}-12 \beta}\right)$ and $\left(-\alpha+\sqrt{\alpha^{2}-12 \beta}\right)$ expressions are negative. Thus the system has no real critical point, indicating that the system has no oscillating solution. However, similar to some of the cases described in the previous subsections, it is possible to have a single bounce if both the energy density and the derivative of hubble parameter at bounce be positive. By tacking into account these parameters at bounce, we have

$\rho_{b}=-\frac{3\left(\zeta_{b}^{4}-\alpha \zeta_{b}^{2}-\beta\right)}{\zeta_{b}^{6}},\left.\quad \frac{d \chi}{d t}\right|_{t_{b}}=\frac{\left(\zeta_{b}^{4}+\alpha \zeta_{b}^{2}+3 \beta\right)}{2 \zeta_{b}^{2}\left(\zeta_{b}^{4}-2 \alpha \zeta_{b}^{2}-3 \beta\right)}$

where positive energy density implies the negative $\left(\zeta_{b}^{4}-\right.$ $\left.\alpha \zeta_{b}^{2}-\beta\right)$, and automatically the negative $\zeta_{b}^{4}-2 \alpha \zeta_{b}^{2}-3 \beta$. Hence the positive $\left.\frac{d \chi}{d t}\right|_{t_{b}}$ requires the negative $\left(\zeta_{b}^{4}+\alpha \zeta_{b}^{2}+\right.$ $3 \beta$ ) which it is impossible for positive values of $\alpha$ and $\beta$, so that there is no bouncing solution to this case. One can see that the cases of $\alpha=$ positive, $\beta=0$ and $\alpha=0, \beta=$ positive are the special forms of $\alpha=$ positive, $\beta=$ positive with no bouncing solution.

The system has two critical points, $P_{1}$ and $P_{2}$ for $\alpha=$ negative, $\beta=$ negative. The energy density at bounce would be

$\rho_{b}=-\frac{3\left(\zeta_{b}^{4}-\alpha \zeta_{b}^{2}-\beta\right)}{\zeta_{b}^{6}}$

where for negative values of $\alpha$ and $\beta$ is negative. This means that without the aforesaid conditions a single bounce never can occur. From Eq. (16), one can also understand this by noting the fact that the parameter $\Omega$ is positive and says that the eigenvalues are real and no oscillating solution can exist. In addition, there are two of the above critical points, $P_{1}\left(\chi_{c}=\right.$ $\left.0, \zeta_{c}=\sqrt{\alpha}\right)$ and $P_{2}\left(\chi_{c}=0, \zeta_{c}=-\sqrt{\alpha}\right)$ for the $\alpha=$ negative, $\beta=$ positive case and also two critical points, $P_{1}\left(\chi_{c}=0, \zeta_{c}=\sqrt{\alpha}\right)$ and $P_{2}\left(\chi_{c}=0, \zeta_{c}=-\sqrt{\alpha}\right)$ for $\alpha=$ negative, $\beta=0$. Here $\Omega=\alpha^{4}$ is positive and indicates that the eigenvalues are real and no oscillating solution can exist.

\section{Conclusion}

The beginning of the universe in the context of general relativity without encountering singularities is not possible, as shown using the singularity theorem if a certain condition is satisfied. Oscillating universes have been explored to solve some problems of the standard cosmological model to avoid the big bang singularity and replace it with a cyclic evolution. Because of that, the outcome of bouncing models is very dependent on the choice of perturbation mechanisms in the background displaying a bounce; the aim of some specific models can be useful for extracting characteristics of a general expected behavior.

Since it is expected that by inclusion of quantum corrections, therefore, the singularity problem be replaced by a quantum bounce; and also with cognizance of the thermodynamical area law that provides an important viability test for any theory of quantum gravity. The aim of this paper was twofold: exploring the bouncing solutions for modified Friedmann equations in order to find out the quantum entropycorrected effects on the metric of FRW universe, and possible signatures of its unknown parameters ( $\alpha$ and $\beta$ ) in satisfying the expected oscillatory evolutions (for matter dominated universes). At this point, from the consensus viewpoint, the value of the logarithmic pre-factor $(\alpha)$ is a point of notable controversy which is constrained to be a negative (from the considerations of LQG) or positive (employed by statistical arguments) value and/or the "best guess" might simply be zero.

By virtue of this matter, there is only the possibility of bouncing-oscillating solutions for curved universes $(k \neq 0)$. In this right, there is the possibility of oscillating solutions and a single bounce (a bouncing evolution without regular repetition) under some appropriate conditions for positive curvature $k=1$ universe with positive $\alpha$ and negative $\alpha$, respectively (with any given value of $\beta$ ); whereas a nonsingular solution does not exist for the case of $k=1$ universe with $\alpha=0$ (and $\beta=0$ ). Furthermore, the possibility of having the oscillating solutions there is only for positive $\alpha$ (and negative $\beta$ ) of a negative curvature $k=-1$ universe. As a result of what was said, accordingly, since the cyclic evolutions should be appeared in discussing the quantum corrections to Friedmann equations; we can emphasize the necessity of non-zero $\alpha$ and by the way recommend the positive values of $\alpha$ for the possibility of having the oscillating solutions and negative values of $\alpha$ for the presence of a quantum bounce. In addition to this result, a detailed description of calculations and how to find the stability analysis of systems was performed in the paper, where illustrates the direction of handling a modified gravity theory along with phase plane analysis in discussing the bouncing evolution of cyclic cosmology scenario.

Open Access This article is distributed under the terms of the Creative Commons Attribution 4.0 International License (http://creativecomm ons.org/licenses/by/4.0/), which permits unrestricted use, distribution, and reproduction in any medium, provided you give appropriate credit to the original author(s) and the source, provide a link to the Creative Commons license, and indicate if changes were made.

Funded by $\mathrm{SCOAP}^{3}$. 


\section{References}

1. S.W. Hawking, R. Penrose, Proc. R. Soc. Lond. A 314, 529 (1970)

2. G.F.R. Ellis, A. King, Commun. Math. Phys. 38, 119 (1974)

3. G.F.R. Ellis, B.G. Schmidt, Gen. Relat. Grav. 8, 915 (1977)

4. C.J.S. Clarke, A. Krolak, J. Geophys. 2, 127 (1986)

5. R.H. Brandenberger, C. Vafa, Nucl. Phys. B 316, 391 (1989)

6. R.H. Brandenberger, Class. Quant. Grav. 28, 204005 (2011). arXiv: 1105.3247

7. G.F.R. Ellis, R. Maartens, Class. Quant. Grav. 21, 223 (2004). arXiv:gr-qc/0211082

8. G.F.R. Ellis, J. Murugan, C.G. Tsagas, Class. Quant. Grav. 21, 233 (2004). arXiv:gr-qc/0307112

9. E. Berti, E. Barausse, V. Cardoso et al., Class. Quant. Grav. 32 $243001(2015)$

10. P. Pavlovic, M. Sossich, Phys. Rev. D 95, 103519 (2017)

11. A. Linde, Lect. Notes Phys. 738, 1 (2008)

12. V.G. Gurzadyan, R. Penrose, Eur. Phys. J. Plus 128, 22 (2013)

13. A.D. Linde, Phys. Lett. B 108, 389 (1982)

14. A.H. Chamseddine, V. Mukhanov, JCAP 1703, 009 (2017)

15. V. Mukhanov, R. Brandenberger, Phys. Rev. Lett. 68, 1969 (1992)

16. V. Mukhanov, R. Brandenberger, A. Sornborger, Phys. Rev. D 48, 1629 (1993)

17. T. Clifton, P.G. Ferreira, A. Padilla, C. Skordis, Phys. Rep. 513, 1 (2012)

18. S. Nojiri, S.D. Odintsov, Phys. Rep. 505, 59 (2011)

19. Y. Misonoh, K. Maeda, T. Kobayashi, Phys. Rev. D 84, 064030 (2011)

20. M. Novello, S.E.P. Bergliaffa, Phys. Rep. 463, 127 (2008)

21. D.J. Mulryne, N.J. Nunes, R. Tavakol, J.E. Lidsey, Int. J. Mod. Phys. A 20, 2347 (2005)

22. T. Thiemann, Lect. Notes Phys. 631, 41 (2003)

23. C. Rovelli, Living Rev. Relativ. 1, 1 (1998)

24. M. Bojowald, Living Rev. Relativ. 8, 11 (2005)

25. M.J. Rees, Observatory 89, 193 (1969)

26. A.A. Starobinsky, JETP Lett. 30, 719 (1979)

27. J. Khoury, B.A. Ovrut, P.J. Steinhardt, N. Turok, Phys. Rev. D 64, $123522(2001)$

28. P.J. Steinhardt, N. Turok, Science 296, 1436 (2002)

29. J. Khoury, B.A. Ovrut, N. Seiberg, P.J. Steinhardt, N. Turok, Phys. Rev. D 65, 086007 (2002)

30. P.J. Steinhardt, N. Turok, Phys. Rev. D 65, 126003 (2002)

31. J. Khoury, P.J. Steinhardt, N. Turok, Phys. Rev. Lett. 91, 161301 (2003)

32. J. Khoury, P.J. Steinhardt, N. Turok, Phys. Rev. Lett. 92, 031302 (2004)

33. Y.-S. Piao, Y.-Z. Zhang, Nucl. Phys. B 725, 265 (2005)

34. Y.-S. Piao, Phys. Rev. D 70, 101302 (2004)

35. Y.-S. Piao, Phys. Lett. B 677, 1 (2009)

36. P.W. Graham, B. Horn, S. Kachru, S. Rajendran, G. Torroba, JHEP 02, 029 (2014). arXiv: 1109.0282

37. A. Salehi, Phys. Rev. D 94, 123519 (2017)

38. E. Alesci, G. Botta, F. Cianfrani, S. Liberati, Phys. Rev. D 96 , 046008 (2017)

39. S.W. Hawking, Commun. Math. Phys. 43, 199 (1975)

40. J.D. Bekenstein, Phys. Rev. D 7, 2333 (1973)

41. G. 't Hooft, Conf. Proc. C 930308, 284-296 (1993). arXiv:gr-qc/9310026

42. L. Susskind, J. Math. Phys. 36, 6377 (1995)

43. T. Jacobson, Phys. Rev. Lett. 75, 1260 (1995)
44. R.G. Cai, Nucl. Phys. B 628, 375 (2002)

45. R.G. Cai, Phys. Lett. B 525, 331 (2002)

46. A.V. Frolov, L. Kofman, JCAP 0305, 009 (2003)

47. R.G. Cai, S.P. Kim, JHEP 0502, 050 (2005)

48. R.G. Cai, L.M. Cao, Y.P. Hu, JHEP 0808, 090 (2008)

49. M. Akbar, R.G. Cai, Phys. Rev. D 7, 084003 (2007)

50. T. Padmanabhan, Rep. Prog. Phys. 73, 046901 (2010)

51. U.H. Danielsson, Phys. Rev. D 71, 023516 (2005)

52. D. Battefeld, P. Peter, Phys. Rep. 571, 1 (2015)

53. A.R. Amani, Int. J. Mod. Phys. D 25, 1650071 (2016)

54. Y.F. Cai et al., Class. Quant. Grav. 28, 215011 (2011)

55. G. Kane, K. Sinha, S. Watson, Int. J. Mod. Phys. D 24, 1530022 (2015)

56. Y. Tavakoli, J.C. Fabris, Int. J. Mod. Phys. D 24, 1550062 (2015)

57. S. Carlip, Class. Quant. Grav. 17, 4175 (2000)

58. G.A. Vilkovisky, Class. Quant. Grav. 9, 895 (1992)

59. K.S. Stelle, Phys. Rev. D 16, 953 (1977)

60. S. Das, P. Majumdar, R.K. Bhaduri, Class. Quant. Grav. 19, 2355 (2002)

61. A. Chatterjee, Phys. Rev. Lett. 92, 141301 (2004)

62. A. Dabholkar, Phys. Lett. B 347, 222 (1995)

63. H.K. Jassal, J.S. Bagla, T. Padmanabhan, MNRAS 356, 11 (2005)

64. A. Ashtekar, T. Pawlowski, P. Singh, Phys. Rev. D 74, 084003 (2006)

65. C. Wetterich, Phys. Lett. B 594, 17 (2004)

66. R.B. Mann, S.N. Solodukhin, Nucl. Phys. B 523, 293 (1998)

67. C. Rovelli, Phys. Rev. Lett. 77, 3288 (1996)

68. J.Z. Ma, X. Zhang, Phys. Lett. B 699, 233 (2011)

69. S. Mukherji, S.S. Pal, JHEP 0205, 026 (2002)

70. R. Banerjee, S. Gangopadhyay, S.K. Modak, Phys. Lett. B 686, $181(2010)$

71. S. Chakraborty, Fundam. Theor. Phys. 187, 43 (2017)

72. E. Papantonopoulos, The effects of curvature correction terms on brane cosmology (2004). arXiv:gr-qc/0402115

73. D.N. Page, New J. Phys. 7, 203 (2005)

74. S.N. Solodukhin, Phys. Rev. D 57, 2410 (1998)

75. J. Zhang, Phys. Lett. B 668, 353 (2008)

76. R. Banerjee, B.R. Majhi, Phys. Lett. B 662, 62 (2008)

77. R.K. Kaul, P. Majumdar, Phys. Rev. Lett. 84, 5255 (2000)

78. M. Domagala, J. Lewandowski, Class. Quant. Grav. 21, 5233 (2004)

79. G. Gour, Phys. Rev. D 66, 104022 (2002)

80. A. Chatterjee, P. Majumdar, Black hole entropy: quantum versus thermal fluctuations (2003). arXiv:gr-qc/0303030

81. S. Hod, Class. Quant. Grav. 21, 97 (2004). (letter to the editor)

82. S.S. More, Class. Quant. Grav. 22, 4129 (2005)

83. K.A. Meissner, Class. Quant. Grav. 21, 5245 (2004)

84. S. Das, R. Kaul, P. Majumdar, Phys. Rev. D 63, 044019 (2001)

85. A. Ghosh, P. Mitra, Phys. Rev. D 71, 027502 (2005)

86. A.J.M. Medved, Class. Quant. Grav. 22, 133 (2005)

87. P. Mitra, Phys. Rev. D 85, 104025 (2012)

88. A. Sheykhi, M.H. Dehghani, S.E. Hosseini, JCAP 04, 038 (2013)

89. A. Sheykhi, Eur. Phys. J. C 69, 265 (2010)

90. A.C. Pope et al., Astrophys. J. 607, 655 (2004)

91. D.J. Eisenstein et al., Astrophys. J. 633, 560 (2005)

92. A.G. Riess et al., Astrophys. J. 116, 1009 (1998)

93. C. Molina-Paris, M. Visser, Phys. Lett. B 455, 90 (1999)

94. R. Durrer, J. Laukenmann, Class. Quant. Grav. 13, 1069 (1996)

95. H. Farajollahi, A. Salehi, Phys. Rev. D 83, 124042 (2011)

96. M. Visser, Science 276, 88 (1997)

97. M. Visser, Phys. Rev. D 56, 7578 (1997) 